\title{
Behavior of Temperature Dependence of $\chi$ Parameter in Random Copolymer Blends Showing an Immiscibility Window
}

\author{
Tsukasa Sato, Masato Suzuki, Masao Tohyama, Masaki Endo, \\ Tomoo SHIomi, ${ }^{\dagger}$ and Kiyokazu IMAI \\ Department of Materials Science and Technology, Nagaoka University of Technology, \\ 1603-1 Kamitomioka, Nagaoka, Niigata 940-21, Japan
}

(Received September 17, 1996)

\begin{abstract}
Previously miscibility for the blends of poly(vinyl chloride-stat-vinyl acetate) (VCVAc 90 , containing $90 \mathrm{wt} \%$ of VC) with poly(isobutyl methacrylate-stat- $n$-butyl methacrylate) (iBMAnBMA) was found to be like a so-called immiscibility window. In this work, the temperature dependence of the Flory-Huggins $\chi$ parameter was calculated for the VCVAc $90 /$ iBMAnBMA blends using the Flory-type equation-of-state theory extended to random copolymer systems. The equation-ofstate parameters necessary for calculation of $\chi$ was determined using the experimental results of osmotic pressures and heats of mixing for solutions of these polymers in cyclohexanone (CHN). The temperature dependence of $\chi$ was a monotonically increasing function over all the copolymer compositions of iBMAnBMA copolymers, and there existed a range of copolymer compositions in which $\chi$ for the copolymer blends VCVAc90/iBMAnBMA was positive though $\chi$ s for both homopolymer blends VCVAc90/PiBMA and VCVAc90/PnBMA (where VCVAc90 was regarded as a homopolymer) were negative. This corresponds qualitatively to miscibility behavior observed for the present copolymer blends. It was concluded that miscibility behavior in the present copolymer blends is one of two types of immiscibility windows classified previously by us.

KEY WORDS Immiscibility Window / Miscibility Window / Random Copolymer Blends / Interaction Parameter $\chi$ / Equation-of-State Theory /
\end{abstract}

Dependence of miscibility on copolymer composition for random copolymer blends has been investigated ${ }^{1-8}$ theoretically and experimentally. In particular, so-called miscibility ${ }^{1-5}$ and immiscibility ${ }^{6,7}$ windows are typical miscibility behavior. The former window is that blends containing random copolymers are miscible in a certain range of the copolymer composition even though any binary combinations of their corresponding homopolymers are immiscible, while the latter window is the reverse case. This miscibility behavior can be explained on the basis of the copolymer composition dependence of the Flory-Huggins intermolecular interaction parameter $\chi$ which is expressed in terms of the intersegmental parameters $\chi_{i j}$ between the different constituent monomers. $^{2-4,7,9}$ Namely, the occurrence of the miscibility and immiscibility windows is based on a change of the sign of intermolecular $\chi$ with copolymer composition. Even though such situations are valid at certain temperatures, they may not be necessarily held at other temperatures because $\chi$ usually depends on temperature.

Equation-of-state theories such as the Flory-Patterson theory ${ }^{10-13}$ and the Sanchez-Lacombe lattice fluid theory ${ }^{14,15}$ have been developed to describe thermodynamic properties including miscibility behavior for polymer blends. For liquid-liquid phase equilibria, neither of the above two theories is superior to the other, as pointed out by Sanchez himself. ${ }^{16}$ An integral equation theory has also been applied to polymer systems recently by Curro and Schweizer. ${ }^{17,18}$ Of these equationof-state approaches, the Flory-Patterson theory based on the principle of corresponding states has been applied to many polymer solutions $\mathrm{s}^{11,19-23}$ and polymer blends ${ }^{24-28}$ because of its practical advantages and simple physical meaning. In this theory, thermodynamic quantities for mixtures have been calculated using the characteristic parameters evaluated from experimental $P-V-T$ relations for the pure components. As long as this evaluation technique is used, as shown previously by Fujisawa et al., ${ }^{29,30}$ all van der Waals-like equation-of-state theories based on the corresponding state principle give almost the same thermodynamic results for polymer solutions at a constant temperature and pressure.

According to the Flory-Patterson equation-of-state theory, ${ }^{10-13}$ there are two kinds of temperature dependence of $\chi:$ a $\mathrm{U}$-shaped curve in which $\chi$ is always positive and a monotonically increasing curve changing from negative to positive with increase in temperature. As described in our previous paper, ${ }^{31}$ each window may be classified into further two types by change of such temperature- $\chi(T-\chi)$ curves with copolymer composition. For immiscibility windows focused on in this work, there are two types of changes of the $T-\chi$ curve with copolymer composition: one is change from a monotonically increasing function of temperature to a Ushaped curve and again to a monotonically increasing function, and the other is a monotonically increasing function regardless of copolymer composition.

Previously we found that the blends of poly(vinyl chloride-stat-vinyl acetate) (VCVAc)/poly(isobutyl methacrylate-stat- $n$-butyl methacrylate) (iBMAnBMA) behave like an immiscibility window. ${ }^{7}$ As shown in Figure 1 , VCVAc with a copolymer composition of $90 \mathrm{wt} \%$ of VC (VCVAc90) was immiscible with iBMAnBMA in a certain range of copolymer composition at a certain temperature although any binary combination of the homopolymers, PiBMA, PnBMA, and VCVAc90, where VCVAc90 is regarded as a homopolymer to avoid com-

\footnotetext{
$\uparrow$ To whom correspondence should be addressed (FAX: +81 (258) 47-9300, e-mail: shiomi@voscc.nagaokaut.ac.jp).
} 


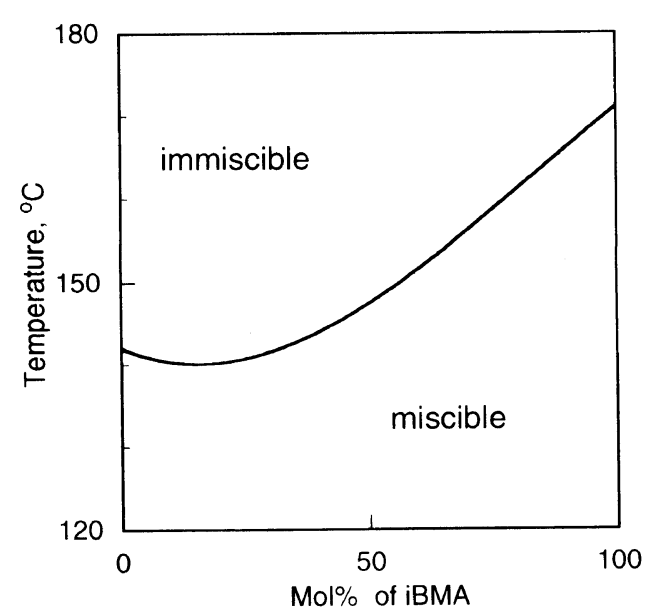

Figure 1. Dependence of miscibility on copolymer composition for VCVAc $90 /$ iBMAnBMA blends at $50 / 50 \mathrm{wt} \%$ blend ratio. ${ }^{7}$

plicated treatment of two kinds of copolymer compositions, was miscible at this temperature. In this work, we evaluated temperature dependence of $\chi$ for these blends showing immiscibility window-like behavior using the Flory equation-of-state theory extended to random copolymer systems, and obtained a change of the $T-\chi$ curve with copolymer composition. On the basis of the results obtained here and simulated in our previous work, ${ }^{31}$ relations between behavior of $T-\chi$ curves and immiscibility windows for the present copolymer blends are discussed. For this purpose, the equation-of-state parameters necessary for calculation of $\chi$ were determined from osmotic pressures and heats of mixing for the binary and ternary solutions of VCVAc90, PiBMA, and PnBMA in cyclohexanone (CHN).

\section{THEORETICAL}

The Flory-type equation-of-state theory ${ }^{10,11}$ starts from a partition function for $N r$-mers with external degrees of freedom of $3 \mathrm{cr}$ per molecule and expresses thermodynamic functions using the characteristic parameters of volume $v^{*}$, pressure $p^{*}$, and temperature $T^{*}$, and their reduced parameters $\tilde{v}\left(=v / v^{*}\right)$ and $\tilde{T}\left(=T / T^{*}\right)$. In the modified Flory equation-of-state theory ${ }^{32-34}$, thermodynamic functions including the interaction parameter $\chi$ for binary mixtures comprising $N_{1} r_{1}$-mers and $N_{2} r_{2}$-mers can be written as follows: The interaction parameter defined by

$$
\begin{aligned}
\chi= & \left(\mu_{1}-\mu_{1}^{0}\right)^{\mathbf{R}} / R T \phi_{2}^{2}=\left(\mu_{1}-\mu_{1}^{0}\right) / R T \phi_{2}^{2} \\
& -\left[\ln \left(1-\phi_{2}\right)+\left(1-r_{1} v_{1}^{*} / r_{2} v_{2}^{*}\right) \phi_{2}\right] / \phi_{2}^{2}
\end{aligned}
$$

is expressed with the residual chemical potential

$$
\begin{aligned}
\left(\mu_{1}-\right. & \left.\mu_{1}^{0}\right)^{\mathrm{R}} \\
= & r_{1} R T\left\{3 \theta_{2}^{2} c_{12} \ln \left[(2 \pi m k T)^{1 / 2} / h\left(g v^{*}\right)^{1 / 3}\left(\tilde{v}^{1 / 3}-1\right)\right]\right. \\
& \left.+3 c_{1} \ln \left(m_{1} / m\right)^{1 / 2}+(3 / 2) c\left(m-m_{1}\right) / m\right\} \\
& +p_{1}^{*} r_{1} v_{1}^{*} \tilde{T}_{1}\left\{\ln \left(v_{1}^{*} / v^{*}\right)+3 \ln \left[\left(\tilde{v}_{1}^{1 / 3}-1\right) /\left(\tilde{v}^{1 / 3}-1\right)\right]\right\} \\
& +2 p^{*} r_{1} v^{*} \tilde{T}\left(v^{*}-\sigma_{1} v_{1}^{*}-\sigma_{2} v_{12}^{*}\right) / v^{*} \\
& +\left(p^{*} r_{1} v^{*} / \tilde{v}\right)\left[\left(\theta_{1} / \sigma_{1}\right)-2\left(v^{*}-\sigma_{1} v_{1}^{*}-\sigma_{2} v_{12}^{*}\right) / v^{*}\right. \\
& \left.-2\left(\theta_{1} p_{1}^{*} v_{1}^{* 2}+\theta_{2} p_{12}^{*} v_{12}^{* 2}\right) /\left(p^{*} v^{* 2}\right)\right]
\end{aligned}
$$

where $\phi_{i}(i=1,2), \theta_{i}$ and $\sigma_{i}$ are the fractions of the core 418 volume, site and segment, respectively. $m$ is the mass of the segment, and $c_{12}$ is deviation from additivity of external degrees of freedom. $g$ is the geometric factor which has been taken as $(4 \pi / 3) 2^{1 / 2}$. The heat of mixing at infinite dilution per gram of component 2 is

$$
\begin{aligned}
& \Delta H_{\mathrm{M}}(\infty)=\left(p_{1}^{*} V_{\mathrm{sp}, 2}^{*} / \tilde{v}_{1}\right)\left(v_{1}^{*} / v_{2}^{*}\right)\left(1+\alpha_{1} T\right)\left[\left(s_{2} / s_{1}\right)\right. \\
& \left.\quad+2\left(v_{12}^{*} / v_{1}^{*}\right)-2\right]+p_{2}^{*} v_{\mathrm{sp}, 2}^{*}\left[\alpha_{1} T\left(T_{1}^{*} / T_{2}^{*}\right) / \tilde{v}_{1}+1 / \tilde{v}_{2}\right] \\
& \quad-2\left(p_{12}^{*} v_{\mathrm{sp}, 2}^{*} / v_{1}\right)\left(v_{1}^{*} / v_{2}^{*}\right)\left(v_{12}^{*} / v_{1}^{*}\right)^{2}\left(s_{2} / s_{1}\right)\left(1+\alpha_{1} T\right) \\
& \quad-R T c_{12}\left(v_{\mathrm{sp}, 2}^{*} / v_{2}^{*}\right)\left(s_{2} / s_{1}\right)\left[(3 / 2)+\alpha_{1} T /\left(\tilde{v}_{1} \tilde{T}_{1}\right)\right]
\end{aligned}
$$

In the derivation of the above $\left(\mu_{1}-\mu_{1}^{0}\right)^{\mathrm{R}}$ and $\Delta H_{\mathrm{M}}(\infty)$, the following combining rules for binary mixture are assumed: $v^{*}$ and $p^{*}$ for the mixture are expressed as

$$
\begin{gathered}
v^{*}=\sigma_{1}^{2} v_{1}^{*}+2 \sigma_{1} \sigma_{2} v_{12}^{*}+\sigma_{2}^{2} v_{2}^{*} \\
v_{12}^{*}=\left[\left(v_{1}^{* 1 / 3}+v_{2}^{* 1 / 3}\right) / 2\right]^{3} \\
p^{*}=\left[\sigma_{1} \theta_{1} p_{1}^{*} v_{1}^{* 2}+\sigma_{2} \theta_{2} p_{2}^{*} v_{2}^{* 2}+2 \sigma_{1} \theta_{2} p_{12}^{*} v_{12}^{* 2}\right] / v^{* 2}
\end{gathered}
$$

where $p_{12}^{*}$ is related to the exchange enthalpy parameter $X_{12}$ by

$$
X_{12}=p_{1}^{*}+\left(v_{2}^{*} / v_{1}^{*}\right)\left(s_{1} / s_{2}\right) p_{2}^{*}-2\left(v_{12}^{*} / v_{1}^{*}\right) p_{12}^{*}
$$

The external degrees of freedom $c$ for the mixture are assumed to be

$$
c=\sigma_{1} c_{1}+\sigma_{2} c_{2}-\sigma_{1} \theta_{2} c_{12}
$$

The characteristic temperature $T^{*}$ can be obtained from eq 8 using the following relation:

$$
p^{*} v^{*}=c R T^{*}
$$

Therefore, thermodynamic functions can be calculated using the characteristic parameters for component polymers and intermolecular parameters such as the surface ratio of segments $s_{2} / s_{1}$, exchange enthalpy parameter $X_{12}$ and deviation from additivity of external degrees of freedom $c_{12}$.

In the systems containing random copolymers, ${ }^{35}$ the characteristic parameters for random copolymers and the intermolecular parameters can be obtained using the characteristic parameters for components of copolymers and the intersegmental parameters, $s_{\mathrm{B}} / s_{\mathrm{A}}, X_{\mathrm{AB}}$, and $c_{\mathrm{AB}}$. For the characteristic parameters of copolymer $2,\left(A_{x}\right.$. $\left.B_{1-x}\right)$, the following combining rules are given on the assumption of random mixing of the segments:

$$
\begin{gathered}
v_{2}^{*}=x^{2} v_{\mathrm{A}}^{*}+2 x(1-x) v_{\mathrm{AB}}^{*}+(1-x)^{2} v_{\mathrm{B}}^{*} \\
p_{2}^{*}=\left[x \theta_{2 \mathrm{~A}} p_{\mathrm{A}}^{*} v_{\mathrm{A}}^{* 2}+(1-x) \theta_{2 \mathrm{~B}} p_{\mathrm{B}}^{*} v_{\mathrm{B}}^{* 2}+2 x \theta_{2 \mathrm{~B}} p_{\mathrm{AB}}^{*} v_{\mathrm{AB}}^{* 2}\right] / v_{2}^{* 2}
\end{gathered}
$$

where

$$
v_{\mathrm{AB}}^{*}=\left[\left(v_{\mathrm{A}}^{* 1 / 3}+v_{\mathrm{B}}^{* 1 / 3}\right) / 2\right]^{3}
$$

and $p_{\mathrm{AB}}^{*}$ can be related to $X_{\mathrm{AB}}$ by

$$
X_{\mathrm{AB}}=p_{\mathrm{A}}^{*}+\left(v_{\mathrm{B}}^{*} / v_{\mathrm{A}}^{*}\right)\left(s_{\mathrm{A}} / s_{\mathrm{B}}\right) p_{\mathrm{B}}^{*}-2\left(v_{\mathrm{AB}}^{*} / v_{\mathrm{A}}^{*}\right) p_{\mathrm{AB}}^{*}
$$

For the external degrees of freedom

$$
c_{2}=x c_{\mathrm{A}}+(1-x) c_{\mathrm{B}}-x \theta_{2 \mathrm{~B}} c_{\mathrm{AB}}
$$

A copolymer effect, i.e., bonding effect between the segments, can be taken into account by the external degrees of freedom $c_{2}$. The characteristic temperature $T_{2}^{*}$ can be given by eq 14 and the following equation: 


$$
p_{2}^{*} v_{2}^{*}=c R T_{2}^{*}
$$

For the mixture of homopolymer 1 and copolymer 2 , the intermolecular parameters $p_{12}^{*}$ and $c_{12}$ in eq 7 and 8 , respectively, can be placed by

$$
\begin{gathered}
p_{12}^{*}=\left[\theta_{2 \mathrm{~A}} p_{1 A}^{*} v_{1 \mathrm{~A}}^{* 2}+\theta_{2 \mathrm{~B}} p_{1 \mathrm{~B}}^{*} v_{1 \mathrm{~B}}^{* 2}\right] / v_{12}^{* 2} \\
c_{12}=\theta_{2 \mathrm{~A}} c_{1 \mathrm{~A}}+\theta_{2 \mathrm{~B}} c_{1 \mathrm{~B}}
\end{gathered}
$$

and $s_{1} / s_{2}$ is expressed as

$$
\mathrm{s}_{1} / s_{2}=s_{1} /\left[x s_{\mathbf{A}}+(1-x) s_{\mathbf{B}}\right]
$$

\section{EXPERIMENTAL}

\section{Materials}

The molecular characteristics for the polymers used in this study are listed in Table I. Poly(isobutyl methacrylate) (PiBMA), poly( $n$-butyl methacrylate) (PnBMA) and poly(isobutyl methacrylate-stat- $n$-butyl methacrylate) (iBMAnBMA) were prepared by radical polymerization in bulk at $80^{\circ} \mathrm{C}$ using $1.3 \mathrm{wt} \%$ of AIBN as an initiator. The conversion was controlled within $20-30 \%$ to avoid copolymer composition drift. The resulting polymers were crudely fractionated using acetone/methanol system. A large second fraction was used for measurements. The polymers obtained were purified by reprecipitating from the acetone solution with a large excess of methanol, and then dried under vacuum. VCVAc copolymer containing $90 \mathrm{wt} \%$ of VC units (VCVAc90) was purchased from Scientific Polymer Products, Inc. (Ontario) and purified by precipitation from THF solution with a large excess volume of petroleum ether.

\section{Measurements}

The interaction parameters $\chi_{\mathrm{p}}$ for ternary solutions containing two kinds of polymers were obtained from osmotic pressures $\pi$ expressed as

$$
\begin{aligned}
-\pi V_{\mathrm{s}} / R T= & \ln \left(1-\phi_{\mathrm{p}}\right)+\left(1-m_{\mathrm{s}} v_{\mathrm{sp}, \mathrm{s}}^{*} / m_{1} v_{\mathrm{sp}, 1}^{*}\right) \phi_{1} \\
& +\left(1-m_{\mathrm{s}} v_{\mathrm{sp}, \mathrm{s}}^{*} / m_{2} v_{\mathrm{sp}, 2}^{*}\right) \phi_{2}+\chi_{\mathrm{p}} \phi_{\mathrm{p}}^{2}
\end{aligned}
$$

where $V_{i}, m_{1}$, and $v_{\mathrm{sp}, i}^{*}$ are the molar volume, mass, and characteristic or core volume per gram, respectively, for component $i$ ( $i=\mathrm{s}, 1$ and 2 indicate the solvent and polymers 1 and 2 , respectively) and $\phi_{\mathrm{p}}$ is the total core volume fraction of polymers given by

$$
\phi_{\mathrm{p}}=\phi_{1}+\phi_{2}=1-\phi_{\mathrm{s}}
$$

The osmotic pressures were measured in a bath ther-

Table I. Characteristics of polymers

\begin{tabular}{lllllll}
\hline \multirow{2}{*}{ Sample } & & & \multicolumn{3}{c}{ Tacticity ${ }^{\mathrm{b}} \%$} \\
\cline { 5 - 6 } & $\bar{M}_{w} \times 10^{-5 \mathrm{a}}$ & $\bar{M}_{w} / \bar{M}_{n}^{\mathrm{a}}$ & & $m m$ & $m r$ & $r r$ \\
\hline VCVAc90 $^{\mathrm{c}}$ & 0.26 & 2.1 & - & - & - \\
PiBMA & 3.0 & 1.7 & 4 & 32 & 64 \\
iBMAnBMA50 & 1.5 & 1.6 & 6 & 36 & 58 \\
PnBMA & 1.9 & 1.7 & 5 & 35 & 60 \\
\hline
\end{tabular}

${ }^{a}$ Determined by GPC measurement, relative to the polystyrene standard. ${ }^{b}$ Determined by ${ }^{13} \mathrm{C}$ NMR measurement in dimethyl sulfoxide (DMSO)- $d_{6} \cdot{ }^{\mathrm{c}}$ Containing $90 \mathrm{wt} \%$ of VC units. ${ }^{\mathrm{d}}$ Containing $50 \mathrm{~mol} \%$ of iBMA units. mostatted at $25+0.03^{\circ} \mathrm{C}$ by a high-pressure osmometer similar to that used by Eichinger and Flory. ${ }^{36}$ Heat of mixing $\Delta H_{\mathrm{M}}(\infty)$ at infinite dilution for glassy polymers was obtained by correcting the heat generated in the microcalorimeter for glass transition. The dissolution process of the glassy polymer in the solvent can be separated into two steps; transition from the glassy state to the rubbery state and then dissolution of the rubbery polymer in the solvent. Therefore, $\Delta H_{\mathrm{M}}(\infty)$ can be expressed by ${ }^{37}$

$$
\Delta H_{\mathrm{M}}(\infty)=\Delta H_{\mathrm{M}}^{\mathrm{app}}(\infty)+\int \Delta C_{\mathrm{p}} \mathrm{d} T
$$

where $\Delta H_{\mathrm{M}}^{\mathrm{app}}(\infty)$ is the heat generated on mixing in the microcalorimeter and $\int \Delta C_{\mathrm{p}} \mathrm{d} T$ is the heat of glass transition obtained from the temperature dependence of specific heats. The calorimeter used was a Tian-Calvet microcalorimeter. Specific heats were determined with a differential scanning calorimeter (DSC) (Seiko I \& E Ltd., DSC 20 with SSC/580 thermal controller and data system) by calibration with sapphire.

\section{RESULTS AND DISCUSSION}

\section{Determination of Characteristic and Intersegmental Pa- rameters}

To calculate thermodynamic functions for mixtures using the equation-of-state theory, we need the characteristic and intermolecular parameters. For the systems containing random copolymers, as described in the theoretical section, the characteristic and intermolecular parameters can be evaluated from those for the copolymer components using eq $10-18$. The values of these parameters for the copolymer components have been assumed to be equivalent to those for the corresponding homopolymers. To calculate $\chi$ parameters for the VCVAc90/ iBMAnBMA blends, therefore, we need the characteristic parameters for VCVAc90, iBMA, and nBMA and the intermolecular or intersegmental parameters for VCVAc90/iBMA, VCVAc90/nBMA, and iBMA/nBMA. Since the characteristic parameters for these polymers and the intersegmental parameter for $\mathrm{iBMA} / \mathrm{nBMA}$ were determined in our previous work, ${ }^{35,38,39}$ as shown in Tables II and III, respectively, we have only to determine

\begin{tabular}{|c|c|c|c|c|}
\hline \multirow{2}{*}{ Component } & $v_{\mathrm{sp}}^{*}$ & $v^{*}$ & $p^{*}$ & $T^{*}$ \\
\hline & $\mathrm{cm}^{3} \mathrm{~g}^{-1}$ & $\mathrm{~cm}^{3} \mathrm{seg} \cdot \mathrm{mol}^{-1}$ & $\mathbf{J ~ \mathrm { cm } ^ { - 3 }}$ & $\mathrm{K}$ \\
\hline $\mathrm{CHN}^{\mathrm{a}}$ & 0.8652 & 68.63 & 628 & 5534 \\
\hline $\mathrm{PiBMA}^{\mathrm{b}}$ & 0.7979 & 62.29 & 584 & 6566 \\
\hline $\mathrm{PnBMA}^{\mathrm{a}}$ & 0.8103 & 66.73 & 577 & 6947 \\
\hline VCVAc $90^{c}$ & 0.6311 & 71.17 & 606 & 7774 \\
\hline iBMAnBMA $50^{d}$ & 0.8041 & 64.44 & 583 & 5678 \\
\hline
\end{tabular}
the intersegmental parameters for VCVAc90/iBMA and VCVAc90/nBMA. These parameters were determined from the experimental results of $\chi_{p}$ for the ternary solutions in $\mathrm{CHN}$ in the way described below. In the present work, copolymer VCVAc90 was dealt with as a

Table II. Characteristic parameters of polymers and solvent obtained at $25^{\circ} \mathrm{C}$

${ }^{\mathrm{a}}$ Reference $38{ }^{\mathrm{b}}$ Reference 39 $^{\mathrm{c}}$ Reference 35 . ${ }^{\mathrm{d}}$ Calculated using the combining rules for random copolymers. 
Table III. Intermolecular or intersegmental parameters

\begin{tabular}{|c|c|c|c|}
\hline System $(i / j)$ & $s_{j} / s_{i}$ & $X_{i j} / \mathbf{J ~ \mathrm { cm } ^ { - 3 }}$ & $c_{i j}$ \\
\hline CHN/VCVAc90 & 0.700 & -32.4 & 0.032 \\
\hline CHN/PiBMA ${ }^{\mathrm{a}}$ & 1.050 & 18.0 & -0.0024 \\
\hline $\mathrm{CHN} / \mathrm{PnBMA}{ }^{\mathrm{b}}$ & 1.100 & 9.93 & 0.0011 \\
\hline VCVAc90/PiBMA & 1.500 & -23.0 & 0.015 \\
\hline VCVAc90/iBMAnBMA50 & 1.535 & -9.30 & 0.0052 \\
\hline VCVAc $90 /$ iBMAnBMA13 ${ }^{c}$ & 1.562 & -0.0468 & 0.00283 \\
\hline VCVAc90/PnBMA & 1.571 & -7.25 & 0.0010 \\
\hline PiBMA/PnBMA ${ }^{\mathrm{b}}$ & 1.048 & 0.0001 & 0.010 \\
\hline
\end{tabular}

${ }^{a}$ Reference $39 .{ }^{b}$ Reference $38 .{ }^{\text {c }}$ Calculated using the combining rules for random copolymers. Numeral values indicate copolymer compositions of iBMA in $\mathrm{mol} \%$.

Table IV. Osmotic pressures $\pi$ and

interaction parameters $\chi_{\mathrm{p}}$ at $25^{\circ} \mathrm{C}^{\mathrm{a}}$

\begin{tabular}{|c|c|c|c|}
\hline$w_{\mathrm{p}}$ & $\phi_{\mathbf{p}}$ & $\pi / \mathrm{J} \mathrm{cm}^{-3}$ & $\chi_{\mathrm{p}}$ \\
\hline & \multicolumn{3}{|c|}{ Binary system } \\
\hline & \multicolumn{3}{|c|}{$\mathrm{CHN} / \mathrm{VCVAc} 90$} \\
\hline 0.0600 & 0.0446 & 0.0238 & 0.044 \\
\hline 0.0836 & 0.0625 & 0.0461 & 0.0497 \\
\hline 0.104 & 0.0778 & 0.0709 & 0.0544 \\
\hline 0.120 & 0.0906 & 0.0928 & 0.0731 \\
\hline \multirow[t]{2}{*}{0.144} & 0.109 & 0.139 & 0.0617 \\
\hline & \multicolumn{3}{|c|}{$\begin{array}{c}\text { Ternary system } \\
\text { CHN/VCVAc } 90 / \text { PiBMA }\end{array}$} \\
\hline 0.0802 & 0.0676 & 0.0256 & 0.302 \\
\hline 0.117 & 0.0989 & 0.0577 & 0.297 \\
\hline 0.154 & 0.131 & 0.108 & 0.291 \\
\hline \multirow[t]{2}{*}{0.181} & 0.156 & 0.158 & 0.292 \\
\hline & \multicolumn{3}{|c|}{ CHN/VCVAc90/iBMAnBMA50 } \\
\hline 0.0671 & 0.0496 & 0.0159 & 0.266 \\
\hline 0.0684 & 0.0494 & 0.0198 & 0.251 \\
\hline 0.104 & 0.0770 & 0.0427 & 0.238 \\
\hline 0.114 & 0.0843 & 0.0530 & 0.229 \\
\hline 0.131 & 0.0974 & 0.0681 & 0.243 \\
\hline 0.151 & 0.113 & 0.102 & 0.217 \\
\hline \multirow[t]{2}{*}{0.182} & 0.138 & 0.154 & 0.218 \\
\hline & \multicolumn{3}{|c|}{ CHN/VCVAc90/PnBMA } \\
\hline 0.0786 & 0.0663 & 0.0230 & 0.317 \\
\hline 0.125 & 0.106 & 0.0618 & 0.317 \\
\hline 0.148 & 0.126 & 0.0909 & 0.314 \\
\hline 0.187 & 0.161 & 0.163 & 0.301 \\
\hline
\end{tabular}

${ }^{\mathrm{a}} w_{\mathrm{p}}$ and $\phi_{\mathrm{p}}$ are the weight and core volume fractions of the polymer, respectively.

homopolymer because only the effect of the copolymer composition dependence of iBMAnBMA was focused on. Thermodynamic treatment of VCVAc as a copolymer had been described in our previous papers. ${ }^{35,40}$

Experimental $\chi_{\mathrm{p}}$ for the solutions in $\mathrm{CHN}$ obtained from the osmotic pressures at $25^{\circ} \mathrm{C}$ by use of eq 19 are summarized in Table IV and plotted against the core volume fraction of polymer in Figure 2. Here, the ratio of the two polymers in the ternary solutions is $1 / 1$ by weight. The heat of mixing $\Delta H_{\mathrm{M}}(\infty)$ at infinite dilution for the VCVAc90 solution in $\mathrm{CHN}$ was obtained at $31{ }^{\circ} \mathrm{C}$ by correction for the heat of glass transition using eq 21 , on the approximation that the same amount of heat as liberated in glass-to-rubber transition was generated on mixing the glassy polymer with the solvent. Figure 3 shows a specific heat curve for VCVAc90 obtained by DSC measurement. The heat of glass transition $\int \Delta C_{\mathrm{p}} \mathrm{d} T$ was evaluated from the area surrounded by two dotdashed lines and the specific heat curve. $\Delta H_{\mathrm{M}}(\infty)$ is shown
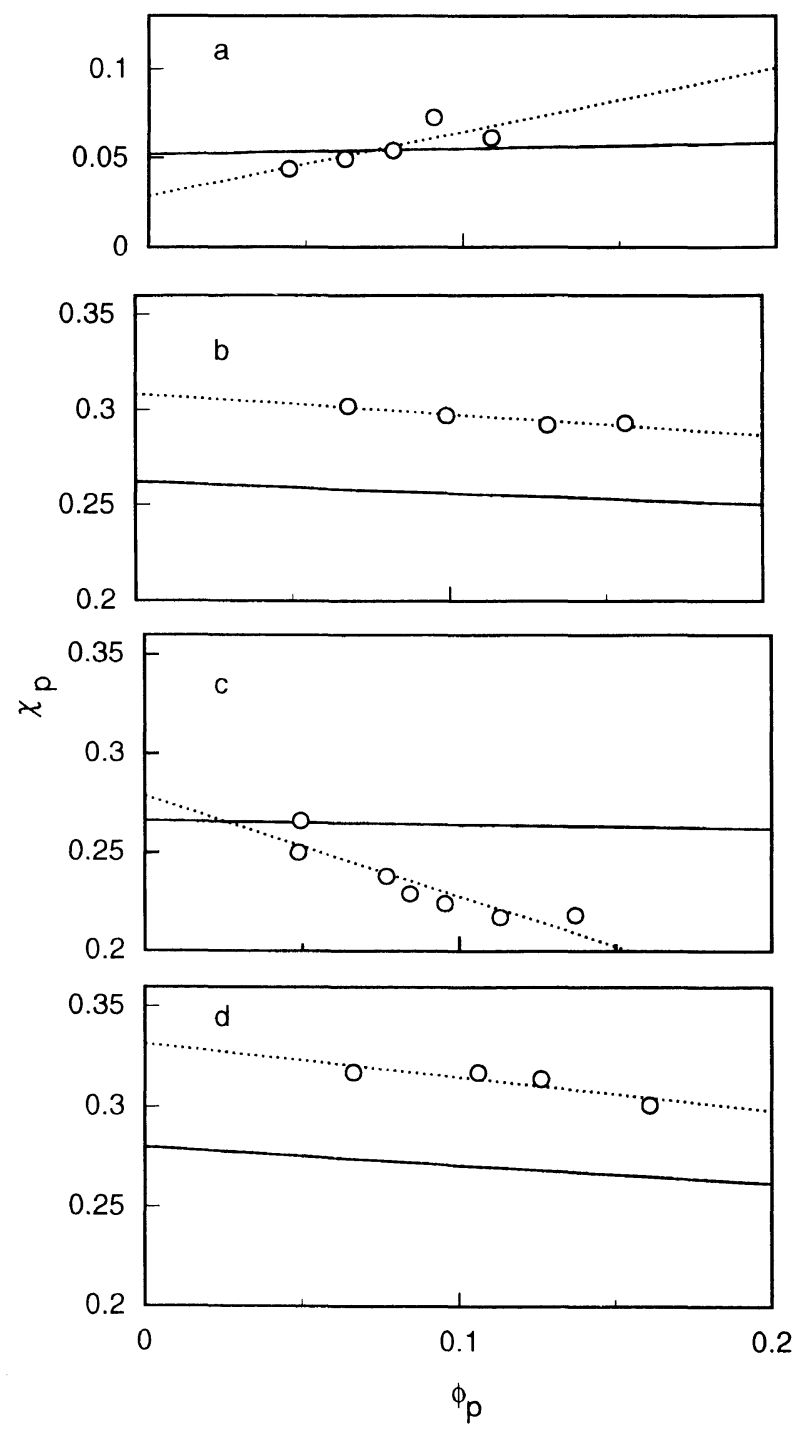

Figure 2. Dependence of $\chi_{\mathrm{p}}$ on core volume fraction $\phi_{\mathrm{p}}$ for polymer solutions in $\mathrm{CHN}$ at $25^{\circ} \mathrm{C}$ : (a) VCVAc90; (b) VCVAc90/PiBMA; (c) VCVAc90/iBMAnBMA50; (d) VCVAc90/PnBMA. The dotted lines are experimental ones, and the solid lines were calculated using the equation-of-state theory, see the text.

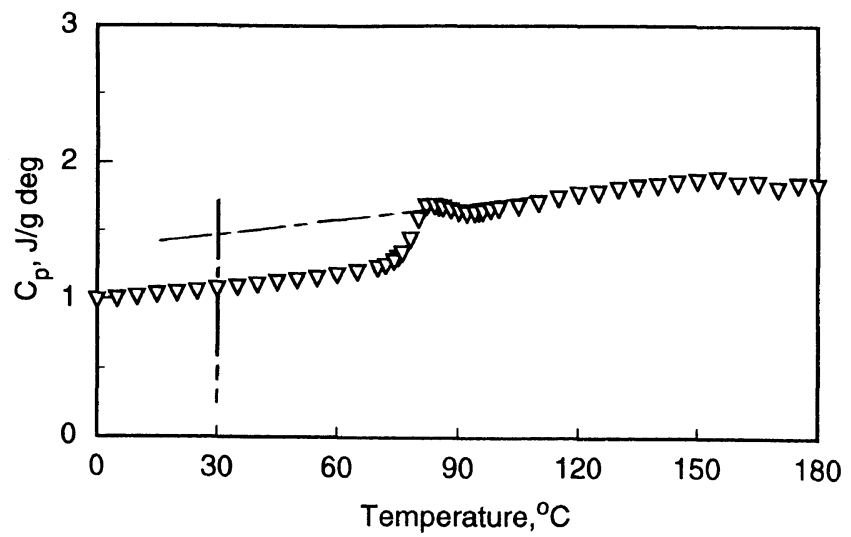

Figure 3. Temperature dependence of the specific heat for VCVAc90.

in Table $\mathrm{V}$ together with $\Delta H_{\mathrm{M}}^{\mathrm{app}}(\infty)$ and $\int \Delta C_{\mathrm{p}} \mathrm{d} T$.

First the intermolecular parameters for $\mathrm{CHN} / \mathrm{VC}$ VAc90 were determined so that the theoretical $\chi$ and $\Delta H_{\mathrm{M}}(\infty)$ could fit the above experimental results. $X_{i j}$ and $c_{i j}$ thus determined for CHN/VCVAc90 are shown in 
Table V. Heat of mixing at infinite dilution at $31^{\circ} \mathrm{C}$

\begin{tabular}{|c|c|c|c|c|}
\hline \multirow{2}{*}{ System } & \multirow{2}{*}{$\frac{\Delta H_{\mathrm{M}}^{\mathrm{app}}(\infty)^{\mathrm{a}}}{\mathrm{Jg}^{-1}}$} & \multirow{2}{*}{$\frac{\int \Delta C_{\mathrm{p}} \mathrm{d} T^{\mathrm{b}}}{\mathbf{J g}^{-1}}$} & \multicolumn{2}{|c|}{$\Delta H_{\mathrm{M}}(\infty), \mathrm{J} \mathrm{g}^{-1}$} \\
\hline & & & $\operatorname{Exptl}^{\mathrm{c}}$ & Calcd $^{\mathrm{d}}$ \\
\hline $\mathrm{CHN} / \mathrm{VCVAc} 90$ & -37.2 & 20.7 & -16.5 & -16.5 \\
\hline
\end{tabular}

${ }^{a}$ Measured at $31{ }^{\circ} \mathrm{C}$ with a Tian-Calvet microcalorimeter. ${ }^{\mathrm{b}}$ Determined from specific heats. ${ }^{\mathrm{c}}$ Obtained by $\Delta H_{\mathrm{M}}^{\mathrm{app}}(\infty)+\int \Delta C_{\mathrm{p}} \mathrm{d} T$. ${ }^{\mathrm{d}}$ Calculated using the equation-of-state theory.

Table III. Here, the surface ratio $s_{j} / s_{i}$ was obtained from the van der Waals radius and surface obtained from the Bondi's table. ${ }^{41}$ The calculated $\chi_{\mathrm{p}}$ and $\Delta H_{\mathrm{M}}(\infty)$ are shown by the solid curve in Figure $2 \mathrm{a}$ and in Table V, respectively. Next the intermolecular or intersegmental parameters, $X_{i j}$ and $c_{i j}$, for VCVAc90/PiBMA and VCVAc90/PnBMA were determined using the results for the ternary solutions. The interaction parameter $\chi_{\mathrm{p}}$ for ternary systems can be expressed as follows using the residual chemical potential: ${ }^{40}$

$$
\begin{aligned}
& \chi_{\mathrm{p}}=\left(\mu_{\mathrm{s}}-\mu_{\mathrm{s}}^{0}\right)^{\mathrm{R}} / R T \phi_{\mathrm{p}}^{2} \\
& \left(\mu_{\mathrm{s}}-\mu_{\mathrm{s}}^{0}\right)^{\mathrm{R}}=r_{\mathrm{s}} R T\left\{3 A \ln \left[(2 \pi m k T)^{1 / 2} / h\left(g v^{*}\right)^{1 / 3}\left(\tilde{v}^{1 / 3}-1\right)\right]\right. \\
& \left.+3 c_{\mathrm{s}} \ln \left(m_{\mathrm{s}} / m\right)^{1 / 2}+(3 / 2) c\left(m-m_{\mathrm{s}}\right) / m\right\} \\
& \left.+p_{\mathrm{s}}^{*} r_{\mathrm{s}} v_{\mathrm{s}}^{*} \tilde{T}_{\mathrm{s}}\left\{\ln \left(v_{\mathrm{s}}^{*} / v^{*}\right)+3 \ln \left[\tilde{v}_{\mathrm{s}}^{1 / 3}-1\right) /\left(\tilde{v}^{1 / 3}-1\right)\right]\right\} \\
& +2 p^{*} r_{\mathrm{s}} v^{*} \tilde{T}(1-W)+p_{\mathrm{s}}^{*} r_{\mathrm{s}} v_{\mathrm{s}}^{*} / \tilde{v}_{\mathrm{s}} \\
& +\left(p^{*} r_{\mathrm{s}} v^{*} / \tilde{v}\right)\left[\left(\theta_{\mathrm{s}} / \sigma_{\mathrm{s}}\right)-2(1-W)-2 Y\right]
\end{aligned}
$$

where

$$
\begin{gathered}
A=\left[\sigma_{\mathrm{s}}\left(1-\theta_{\mathrm{s}}\right) \theta_{1} c_{\mathrm{s} 1}+\sigma_{\mathrm{s}}\left(1-\theta_{\mathrm{s}}\right) \theta_{2} c_{\mathrm{s} 2}-\sigma_{1} \theta_{\mathrm{s}} \theta_{2} c_{12}\right] / \sigma_{\mathrm{s}} \\
W=\left(\sigma_{\mathrm{s}} v_{\mathrm{s}}^{*}+\sigma_{1} v_{\mathrm{s} 1}^{*}+\sigma_{2} v_{\mathrm{s} 2}^{*} / v^{*}\right. \\
Y=\left(\theta_{\mathrm{s}} p_{\mathrm{s}}^{*} v_{\mathrm{s}}^{* 2}+\theta_{1} p_{\mathrm{s} 1}^{*} v_{\mathrm{s} 1}^{* 2}+\theta_{2} p_{\mathrm{s} 2}^{*} v_{\mathrm{s} 2}^{* 2}\right) /\left(p^{*} v^{* 2}\right)
\end{gathered}
$$

Here, the combining rules for ternary systems are given by extension of those for the binary mixture as

$$
\begin{aligned}
v^{*}= & \sigma_{\mathrm{s}}^{2} v_{\mathrm{s}}^{*}+\sigma_{1}^{2} v_{1}^{*}+\sigma_{2}^{2} v_{2}^{*}+2\left(\sigma_{\mathrm{s}} \sigma_{1} v_{\mathrm{s} 1}^{*}+\sigma_{1} \sigma_{2} v_{12}^{*}+\sigma_{\mathrm{s}} \sigma_{2} v_{\mathrm{s} 2}^{*}\right) \\
p^{*}= & {\left[\sigma_{\mathrm{s}} \theta_{\mathrm{s}} p_{\mathrm{s}}^{*} v_{\mathrm{s}}^{* 2}+\sigma_{1} \theta_{1} p_{1}^{*} v_{1}^{* 2}+\sigma_{2} \theta_{2} p_{2}^{*} v_{2}^{* 2}\right.} \\
& \left.+2\left(\sigma_{\mathrm{s}} \theta_{1} p_{\mathrm{s} 1}^{*} v_{\mathrm{s} 1}^{* 2}+\sigma_{1} \theta_{2} p_{12}^{*} v_{12}^{* 2}+\sigma_{\mathrm{s}} \theta_{2} p_{\mathrm{s} 2}^{*} v_{\mathrm{s} 2}^{* 2}\right)\right] / v^{* 2}
\end{aligned}
$$

where $v_{i j}^{*}$ and $p_{i j}^{*}$ are given by the same expressions as eq 5 and 7 , respectively.

$$
c=\sigma_{\mathrm{s}} c_{\mathrm{s}}+\sigma_{1} c_{1}+\sigma_{2} c_{2}-\left(\sigma_{\mathrm{s}} \theta_{1} c_{\mathrm{s} 1}+\sigma_{1} \theta_{2} c_{12}+\sigma_{\mathrm{s}} \theta_{2} c_{\mathrm{s} 2}\right)
$$

$$
p^{*} v^{*}=c R T^{*}
$$

$X_{i j}$ and $c_{i j}$ for VCVAc90/PiBMA and VCVAc90/PnBMA were determined so that the above theoretical $\chi_{\mathrm{p}}$ could fit the experimental ones as well as possible for the three ternary solutions (Figures $2 \mathrm{~b}$, c, and d), using the intermolecular parameters obtained previously ${ }^{38,39}$ for $\mathrm{CHN} /$ PiBMA, CHN/PnBMA and PiBMA/PnBMA and determined above for CHN/VCVAc90. Here, the surface ratio $s_{\text {iBMA }} / s_{\mathrm{VCVAc} 90}$ and $s_{\mathrm{nBMA}} / s_{\mathrm{VCVAc}}$, were obtained by $\left(s_{\text {iBMA }} / s_{\text {CHN }}\right) /\left(s_{\text {VCVAc } 90} / s_{\text {CHN }}\right)$ and $\left(s_{\text {nBMA }} / s_{\text {CHN }}\right) /\left(s_{\text {VCVAc } 90} /\right.$ $\left.s_{\mathrm{CHN}}\right)$. When calculated $\chi_{\mathrm{p}}$ for the two ternary solutions containing homopolymers PiBMA and PnBMA, respectively, are fitted to experiments completely, the calculated ones for solutions of VCVAc90/iBMAnBMA-

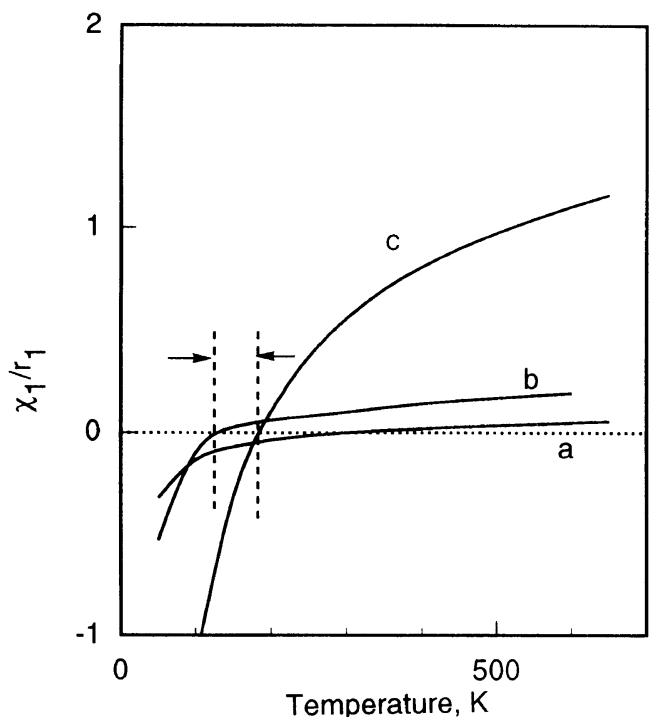

Figure 4. The temperature dependence of $\chi_{1} / r_{1}$ for VCVAc $90 /$ iBMAnBMA blends. Copolymer compositions of iBMA are (a) $0 \mathrm{~mol} \%$, (b) $13 \mathrm{~mol} \%$, and (c) $100 \mathrm{~mol} \%$, respectively.

50 deviate towards larger values. Therefore, $X_{i j}$ and $c_{i j}$ were chosen so that deviations of $\chi_{p}$ from experiments for these three ternary solutions could be smallest as a whole. The values of the intersegmental parameters determined thus are listed in Table III, and the theoretical curves of $\chi_{\mathrm{p}}$ are shown by the solid lines in Figure 2 .

\section{Temperature Dependence of $\chi$ for Copolymer Blends}

Using the characteristic and intersegmental parameters obtained above for VCVAc90, iBMA, and nBMA, we can calculate $\chi$ for VCVAc90/iBMAnBMA copolymer blends. The calculation of the parameter $\chi$ was carried out at $\phi_{2} \rightarrow 0$ to avoid complication of the concentration dependence of $\chi$. Calculated temperature- $\chi_{1} / r_{1}\left(\chi_{1}=\right.$ $\lim _{\phi_{2} \rightarrow 0} \chi$ ) curves were monotonically increasing functions regardless of the copolymer composition of the iBMAnBMA copolymer. Namely the blends of VCVAc90 with the copolymer iBMAnBMA have LCST. In Figure 4 is shown the temperature dependence of $\chi_{1} / r_{1}$ for the homopolymer blends of VCVAc90/PiBMA and VCVAc90/PnBMA and copolymer blend of VCVAc90/iBMAnBMA13 containing $13 \mathrm{~mol} \%$ iBMA units. It should be noted that there is a temperature range, between two dashed lines in Figure 4 , in which $\chi_{1} / r_{1}$ is positive for the copolymer blend while negative for both homopolymer blends. This means that in this temperature range the copolymer blend VCVAc90/iBMAnBMA13 is immiscible though both homopolymer blends VCVAc90/PiBMA and VCVAc90/PnBMA are miscible. In other words, the copolymer blends change from miscible to immiscible and again to miscible with copolymer composition in this temperature range. The trend of this miscibility behavior corresponds to the experimental one shown in Figure 1. Even in use of $X_{\mathrm{ij}}$ and $c_{i j}$ obtained so that the theoretical $\chi_{\mathrm{p}}$ for the ternary solutions containing homopolymers PiBMA and PnBMA, respectively, could fit the experimental one completely, the same behavior was obtained although the copolymer composition range in which the im- 

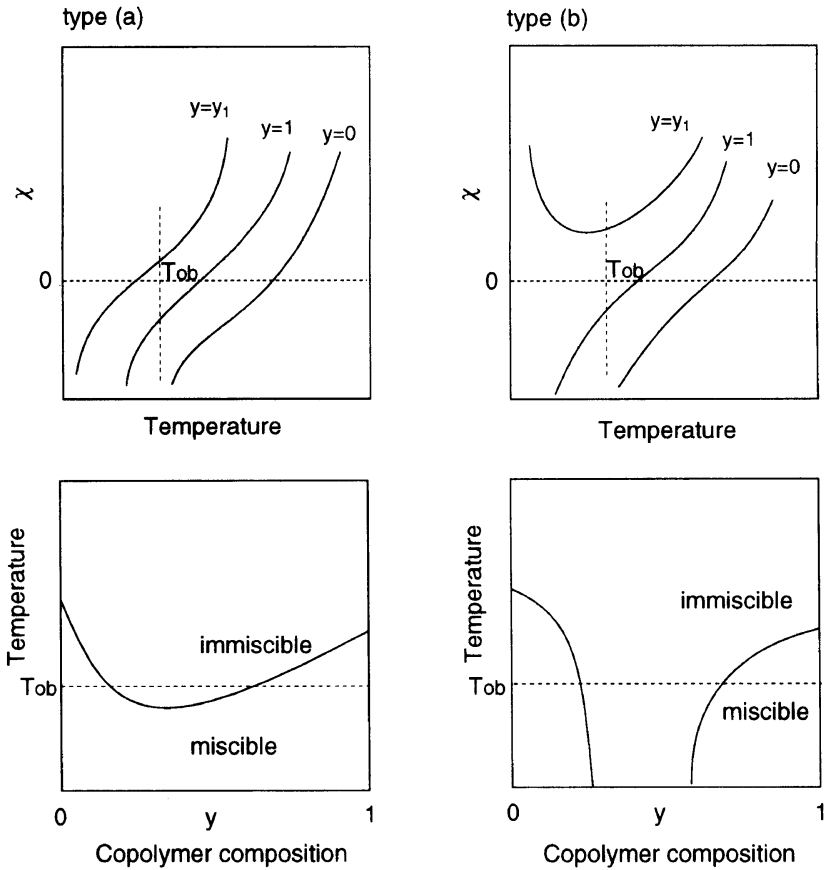

Figure 5. Schematic illustrations of the temperature dependence of $\chi$ (upper) and the corresponding miscibility behavior (lower) for copolymer blends showing immiscibility windows around a temperature $T_{\mathrm{ob}} . y$ indicates the fraction of copolymer composition.

miscibility window appeared shifted. In both calculated results no quantitative agreement was obtained on the boundary temperature of miscibility. One reason may be due to the poor reproducibility of temperature dependence in the Flory-type equation-of-state, as seen by the fact that the characteristic parameters, i.e., reduction parameters, which should be independent of temperature depend on temperature. ${ }^{29}$ In the present study, the values of the characteristic parameters at $25^{\circ} \mathrm{C}$ were used.

As discussed in the previous paper, ${ }^{31}$ there are two changes in the $T-\chi$ curves with copolymer composition for the miscibility behavior like an immiscibility window. In type (a) shown in Figure 5, the $T-\chi$ curves are monotonically increasing functions regardless of copolymer composition. This means that blends are miscible below a certain temperature for any copolymer composition even though the miscibility behavior is like an immiscibility window at a certain temperature $T_{\text {ob}}$. In type (b) the $T-\chi$ curves change from a monotonically increasing function to a U-shaped curve and again to a monotonically increasing function with the copolymer composition. In this case, as shown in the lower figure of Figure 5(b), the blends are immiscible over all temperatures in the copolymer composition range corresponding to U-shaped $T-\chi$ curves. Thus, miscibility behavior of the immiscibility window can be classified into two types. The immiscibility window for the present copolymer blends of VCVAc90/iBMAnBMA is concluded to be type (a) from both results of the miscibility observation in Figure $1^{7}$ and the $T-\chi$ curve behavior obtained in the present work.

\section{REFERENCES}

1. R. Vuković, F. E. Karasz, and W. J. Macknight, Polymer, 24, 529 (1983).

2. R. P. Kambour, J. T. Bendler, and R. C. Bopp, Macromolecules, 16, 753 (1983).

3. G. ten Brinke, F. E. Karasz, and W. J. MacKnight, Macromolecules, 16, 1827 (1983).

4. D. R. Paul and J. W. Barlow, Polymer, 25, 487 (1984).

5. T. Shiomi, F. E. Karasz, and W. J. MacKnight, Macromolecules, 19, 2274, 2644 (1986)

6. A. C. Fernandes, J. W. Barlow, and D. R. Paul, J. Appl. Polym. Sci., 32, 5481 (1986).

7. T. Shiomi, M. Suzuki, M. Tohyama, and K. Imai, Macromolecules, 22, 3578 (1989).

8. T. Sato, M. Endo, T. Shiomi, and K. Imai, Polymer, 37, 2131 (1996).

9. R. Simha and H. Branson, J. Chem. Phys., 12, 253 (1944).

10. P. J. Flory, J. Am. Chem. Soc., 87, 1833 (1965).

11. P. J. Flory, Discuss. Faraday Soc., 49, 7 (1970).

12. D. Patterson and A. Robard, Macromolecules, 11, 690 (1978).

13. D. Patterson, Polym. Eng. Sci., 22, 64 (1982) .

14. I. C. Sanchez and R. H. Lacombe, J. Phys. Chem., 80, 2352 (1976).

15. R. H. Lacombe and I. C. Sanchez, J. Phys. Chem., 80, 2568 (1976).

16. I. C. Sanchez, in "Polymer Compatibility and Incompatibility," K. Solć, Ed., MMI Press, Harwood Academic, New York, 1981, p 59.

17. K. S. Schweizer and J. G. Curro, Phys. Rev. Lett., 60, 809 (1988).

18. J. G. Curro, A. Yethiraj, K. S. Schweizer, J. D. McCoy, and K. G. Honnell, Macromolecules, 26, 2655 (1993).

19. P. J. Flory and H. Shih, Macromolecules, 5, 761 (1972).

20. K. Sugamiya, N. Kuwahara, and M. Kaneko, Macromolecules, 7, 66 (1974).

21. T. Shiomi, Z. Izumi, F. Hamada, and A. Nakajima, Macromolecules, 13, 1149 (1980).

22. T. Shiomi, Y. Kohra, F. Hamada, and A. Nakajima, Macromolecules, 13, 1154 (1980).

23. D. J. Walsh and G. T. Dee, Polymer, 29, 656 (1988).

24. G. ten Brinke, A. Eshuis, E. Roerdink, and G. Challa, Macromolecules, 14, 867 (1981).

25. S. Cimmino, E. Martuscelli, and C. Silvestre, Polymer, 30, 393 (1989).

26. D. J. Walsh, G. T. Dee, J. L. Halary, J. M. Ubiche, M. Millequant, J. Lesec, and L. Monnerie, Macromolecules, 22, 3395 (1989).

27. T. Shiomi, F. Hamada, T. Nasako, K. Yoneda, K. Imai, and A. Nakajima, Macromolecules, 23, 229 (1990).

28. T. Shiomi, F. Hamada, M. Mihoichi, T. Nasako, and A. Nakajima, Polymer, 31, 448 (1990).

29. K. Fujisawa, T. Shiomi, F. Hamada, and A. Nakajima, Polym. J., 13, 993 (1981).

30. K. Fujisawa, T. Shiomi, F. Hamada, A. Nakajima, and J. Lee, Polym. J., 13, 1003 (1981).

31. T. Shiomi and K. Imai, Polymer, 32, 73 (1991).

32. F. Hamada, T. Shiomi, K. Fujisawa, and A. Nakajima, Macromolecules, 13, 729 (1980).

33. T. Shiomi, K. Fujisawa, F. Hamada, and A. Nakajima, J. Chem. Soc., Faraday Trans. 2, 76, 895 (1980).

34. K. Fujisawa, T. Shiomi, F. Hamada, and A. Nakajima, Polym. Bull., 3, 261 (1980).

35. T. Shiomi, H. Ishimatsu, T. Eguchi, and K. Imai, Macromolecules, 23, 4970 (1990)

36. B. E. Eichinger and P. J. Flory, Trans, Faraday Soc., 64, 2035 (1968).

37. G. V. Schulz, K. V. Guenner, and H. Gerrens, Z. Phys. Chem. (F), 4, 192 (1955).

38. T. Shiomi, M. Tohyama, M. Endo, T. Sato, and K. Imai, J. Polym. Sci., Polym. Phys., 34, 2599 (1996).

39. T. Sato, M. Tohyama, M. Suzuki, T. Shiomi, and K. Imai, Macromolecules, 29, 8231 (1996).

40. T. Shiomi, T. Eguchi, H. Ishimatsu, and K. Imai, Macromolecules, 23, 4978 (1990).

41. A. Bondi, J. Phys. Chem., 87, 1833 (1965). 